\title{
Isolation of Natural Fungal Pathogens from Marchantia polymorpha Reveals Antagonism between Salicylic Acid and Jasmonate during Liverwort-Fungus Interactions
}

\author{
Hidenori Matsui ${ }^{1,7,8}$, Hidekazu Iwakawa ${ }^{2,8}$, Gang-Su Hyon ${ }^{1,8}$, Izumi Yotsui ${ }^{1,9}$, Shinpei Katou ${ }^{3,9}$, \\ Isabel Monte ${ }^{4,9}$, Ryuichi Nishihama ${ }^{5}$, Rainer Franzen ${ }^{6}$, Roberto Solano ${ }^{4}$ and Hirofumi Nakagami ${ }^{1,2, *}$ \\ ${ }^{1}$ Plant Proteomics Research Unit, RIKEN CSRS, Yokohama, Kanagawa, 230-0045 Japan \\ ${ }^{2}$ Basic Immune System of Plants, Max-Planck Institute for Plant Breeding Research, 50829 Cologne, Germany \\ ${ }^{3}$ Faculty of Agriculture, Shinshu University, Minamiminowa 8304, Nagano, 399-4598 Japan \\ ${ }^{4}$ Department of Plant Molecular Genetics, Centro Nacional de Biotecnología, Consejo Superior de Investigaciones Científicas (CNB-CSIC), 28049 \\ Madrid, Spain \\ ${ }^{5}$ Graduate School of Biostudies, Kyoto University, Kyoto, 606-8502 Japan \\ ${ }^{6}$ Central Microscopy, Max-Planck Institute for Plant Breeding Research, 50829 Cologne, Germany \\ 7Present address: Graduate School of Environmental and Life Sciences, Okayama University, Okayama, 700-8530 Japan \\ ${ }^{8}$ These authors contributed equally to this work. \\ ${ }^{9}$ These authors contributed equally to this work. \\ *Corresponding author: E-mail, nakagami@mpipz.mpg.de; Fax, +49-221-5062-353. \\ (Received May 24, 2019; Accepted September 15, 2019)
}

The evolution of adaptive interactions with beneficial, neutral and detrimental microbes was one of the key features enabling plant terrestrialization. Extensive studies have revealed conserved and unique molecular mechanisms underlying plantmicrobe interactions across different plant species; however, most insights gleaned to date have been limited to seed plants. The liverwort Marchantia polymorpha, a descendant of early diverging land plants, is gaining in popularity as an advantageous model system to understand land plant evolution. However, studying evolutionary molecular plant-microbe interactions in this model is hampered by the small number of pathogens known to infect M. polymorpha. Here, we describe four pathogenic fungal strains, Irpex lacteus Marchantiainfectious (MI)1, Phaeophlebiopsis peniophoroides MI2, Bjerkandera adusta $\mathrm{MI} 3$ and $B$. adusta $\mathrm{MI} 4$, isolated from diseased M. polymorpha. We demonstrate that salicylic acid (SA) treatment of $M$. polymorpha promotes infection of the I. lacteus MI1 that is likely to adopt a necrotrophic lifestyle, while this effect is suppressed by co-treatment with the bioactive jasmonate in M. polymorpha, dinor-cis-12-oxo-phytodienoic acid (dnOPDA), suggesting that antagonistic interactions between SA and oxylipin pathways during plant-fungus interactions are ancient and were established already in liverworts.

Keywords: Dinor-cis-12-oxo-phytodienoic acid • Irpex lacteus - Marchantia polymorpha - Mpcoi1 - Necrotrophic fungal pathogen - Salicylic acid.

\section{Introduction}

Acquisition and evolution of adaptive mechanisms providing resistance to biotic and abiotic stresses enabled land plants to conquer terrestrial habitats (Delwiche and Timme 2011, Delwiche and Cooper 2015, Rensing 2018). Land plants have coevolved with a large variety of microbes since their colonization of land more than 450 million years ago (Bonfante and Genre 2010, Field et al. 2015, Martin et al. 2017, Field and Pressel 2018, Hoysted et al. 2018, Mathieu et al. 2018, Morris et al. 2018, Spribille 2018, Strullu-Derrien 2018), and it has been hypothesized that mutualistic symbiotic interactions with fungi facilitated plant terrestrialization (Field et al. 2015, Rensing 2018). Given that most microbes are not beneficial to plants and because most plants are resistant to the majority of potentially pathogenic microbes, establishment of a sophisticated immune system probably also played a crucial role in plant terrestrialization (de Vries et al. 2018).

Extant bryophytes, comprising liverworts, hornworts and mosses, are the earliest diverging lineages of land plants, and, as such, represent key plant species for understanding the origin and evolution of molecular systems in plants (Rensing 2017, Puttick et al. 2018). Bryophytes are non-vascular gametophyte dominant plants and develop root-like anchoring structures called rhizoids (Jones and Dolan 2012, Duckett et al. 2014, Harrison 2017). Rhizoids are utilized to take up nutrients and water and also serve as entry sites for symbiotic fungi (Ligrone et al. 2007, Pressel et al. 2008, Humphreys et al. 2010, Pressel et al. 2010, Silvani et al. 2012, Field et al. 2016). Since bryophytes and seed plants have notable differences in their body plans, microbes may have applied different approaches to interact with these plant species. Conversely, bryophytes and seed plants may have followed a unique evolutionary trajectory that has allowed interaction with a large variety of microbes.

Establishment of the moss Physcomitrella patens as a model plant paved the way for the study of evolutionary molecular 
plant-microbe interactions (EvoMPMI), although there is no evidence of mycorrhiza-like associations in mosses (Rensing et al. 2008, Lehtonen et al. 2009, Pressel et al. 2010, Ponce de León 2011, Castro et al. 2016, Ponce de León and Montesano 2017, Upson et al. 2018, Vigneron et al. 2018). Several studies have shown that pathogenic microbes including bacteria, fungi and oomycetes isolated from other plant species can cause disease in P. patens (Andersson et al. 2005, Ponce de León et al. 2007, Oliver et al. 2009, Ponce de León and Montesano 2013, Reboledo et al. 2015, Overdijk et al. 2016). A study on the evolutionarily conserved Lys $M$ receptor kinase CERK1 in $P$. patens demonstrated that the LysM-mediated chitin-triggered immune system was established early in evolution and remained well conserved thereafter (Bressendorff et al. 2016). Regarding natural infection in mosses, the fungal pathogens Irpex sp. and Fusarium sp. were isolated from the moss Racomitrium japonicum, and the mosses Aulacomnium palustre, Hylocomium splendens and Polytrichum juniperinum are native hosts of the fungal pathogen Atradidymella muscivora (Davey et al. 2009, Lehtonen et al. 2009). Further, tobacco mosaic virus or its related virus was detected in the Antarctic mosses Polytrichum and Barbilophozia, and a necrotrophic fungus Scleroconidioma sphagnicola causes disease in the peat moss Sphagnum fuscum (Tsuneda et al. 2001, Polischuk et al. 2007).

The thalloid liverwort Marchantia polymorpha is emerging as another promising bryophyte model system (Berger et al. 2016, Ishizaki et al. 2016, Shimamura 2016, Delwiche et al. 2017), and molecular genetic tools and techniques for $M$. polymorpha are now well established (Ishizaki et al. 2008, Ishizaki et al. 2013, Kubota et al. 2013, Ishizaki et al. 2015, Flores-Sandoval et al. 2016, Nishihama et al. 2016, Sugano et al. 2018). Importantly, M. polymorpha probably did not undergo whole-genome duplication, with the consequence that genetic redundancy is low (Bowman et al. 2017). The overall simplicity of gene families in the species holds advantages for molecular genetic analysis (Eklund et al. 2015, Kato et al. 2015, Lockhart 2015). The majority of immune-related genes characterized in seed plants, including nucleotide-binding leucine-rich repeat (NLR) genes, have been identified in the M. polymorpha genome (Xue et al. 2012, Bowman et al. 2017). However, M. polymorpha has probably lost its symbiotic genes and cannot form beneficial associations with mycorrhizal fungi as in the case of $P$. patens. It is worth noting that most thalloid liverworts including related Marchantia species are able to form mycorrhizal associations (Russell and Bulman 2004, Ligrone et al. 2007, Bidartondo and Duckett 2010, Humphreys et al. 2010, Silvani et al. 2012). The study of plant-microbe interactions in $M$. polymorpha requires the establishment of proper and diverse pathosystems. However, information on pathogenic microbes that cause disease in $M$. polymorpha or liverworts remains very limited (Carella and Schornack 2018). Recently, the hemi-biotrophic oomycete pathogen Phytophthora palmivora, which is virulent for the root and leaf tissues of a broad range of flowering plants, was shown to infect and cause disease in M. polymorpha (Carella et al. 2018). Interestingly, P. palmivora could form haustoria-like intracellular infection structures in $M$. polymorpha as in flowering plants, which was not observed in $P$. patens (Overdijk et al. 2016, Carella et al. 2018). Besides, a large number of fungal endophytes were isolated from wild populations of M. polymorpha (Nelson et al. 2018, Nelson and Shaw 2019). Individual inoculation of axenic M. polymorpha with the isolated fungi suggested that some of the fungi, namely Xylaria cubensis, Hypoxylon submonticulosum and Colletotrichum sp., were rather pathogenic to M. polymorpha.

Plants are subject to attack by various pathogenic microbes with different lifestyles, namely biotrophs, necrotrophs and hemibiotrophs (Glazebrook 2005). Biotrophs derive their nutrients from living host tissues, while necrotrophs feed on the contents of dying or dead cells. Hemi-biotrophs switch their lifestyle from biotrophic to necrotrophic during infection. In seed plants, phytohormones play crucial roles in inducing relevant defense responses against these contrasting pathogens (Thomma et al. 1998, Spoel and Dong 2008, Pieterse et al. 2009, Verhage et al. 2010, Berens et al. 2017). The salicylic acid (SA) pathway primarily induces resistance against biotrophic and hemi-biotrophic pathogens, and the jasmonic acid (JA) pathway is chiefly required for resistance against necrotrophic pathogens and herbivorous insects. Crosstalk between the SA and JA pathways, which can spatiotemporally optimize distinct defense responses, is considered to be a fundamental regulatory mechanism for seed plants to deal with simultaneous or consecutive attack by multiple pathogens in natural environments (Koornneef and Pieterse 2008, Betsuyaku et al. 2018, Tsuda 2018). The SA and JA pathways are typically mutually antagonistic, and this SA-JA antagonism has been observed in various seed plants (Thaler et al. 2012). In Arabidopsis, SA is typically prioritized over JA, and the SA receptor NPR1 acts as a negative regulator of SA-induced suppression of the JA pathway (Spoel et al. 2003, Spoel et al. 2007, Leon-Reyes et al. 2010). Although homologs of core signaling components of the SA and JA pathways, including NPR1 and the JA-receptor COI1, were identified in the genomes of non-seed plant species, there is as yet no evidence for SA-JA crosstalk in non-seed plants (Thaler et al. 2012, Berens et al. 2017).

SA and JA are present in a range of bryophytes, although the detected JA levels were imperceptible (Stumpe et al. 2010, Drábková et al. 2015). Importantly, M. polymorpha and P. patens lack two key enzymes, OPR3 and JAR1, that are needed to produce JA-lle from 12-oxo-phytodienoic acid (OPDA) in vascular plants (Rensing et al. 2008, Ponce de León et al. 2015, Yamamoto et al. 2015, Bowman et al. 2017). It has been consistently shown that $M$. polymorpha does not accumulate the bioactive JA, JA-lle, and M. polymorpha, P. patens, and the hornwort Anthoceros agrestis are insensitive to exogenous application of JA or JA-lle (Yamamoto et al. 2015, Monte et al. 2018). However, bryophytes remain sensitive to exogenous application of OPDA, and the M. polymorpha COI1 homolog MpCOI1 was found to be a receptor for dinor-OPDA (dn-OPDA) isomers, which are also catabolic products of OPDA, but MpCOI1 does not perceive JA-lle (Ponce de León et al. 2012, Ponce de León et al. 2015, Monte et al. 2018). This finding may suggest that dnOPDA isomers play roles in bryophytes that are similar to those of JA-lle in seed plants. P. patens accumulates OPDA upon inoculation with the necrotrophic pathogens, Botrytis cinerea, Pythium debaryanum and Pythium irregulare, and Mpcoi1 mutants displayed reduced resistance against the generalist 
herbivore Spodoptera littoralis, suggesting a role of the OPDACOI1 pathway in defense responses in bryophytes (Oliver et al. 2009, Ponce de León et al. 2012, Monte et al. 2018). However, the contributions of OPDA catabolites and COI1 homologs to resistance against necrotrophic microbes in bryophytes remain uncharacterized. It should be noted that $P$. patens can respond to methyl jasmonate (MeJA) treatment, but it is not clear how $P$. patens perceives MeJA and the biological relevance of this perception remains unknown (Ponce de León et al. 2012, Ponce de León et al. 2015). Regarding the SA pathway in bryophytes, one of the P. patens NPR1 homologs was shown to partially complement the Arabidopsis npr1-1 mutant phenotypes, suggesting that the molecular properties of NPR1 are relatively conserved throughout evolution (Peng et al. 2017). In P. patens, SA pretreatment could induce resistance against the bacterial pathogen Erwinia carotovora (Andersson et al. 2005). However, SA function in defense responses and the role of NPR 1 in the SA pathway in bryophytes remain to be clarified.

Here, we report the isolation of four pathogenic fungal strains from diseased M. polymorpha. The four fungal isolates, Irpex lacteus Marchantia-infectious (MI)1, Phaeophlebiopsis peniophoroides MI2, Bjerkandera adusta MI3 and B. adusta MI4, displayed different levels of pathogenicity against in vitro grown M. polymorpha. Furthermore, by using a newly established I. lacteus MI1-M. polymorpha pathosystem, we provide first evidence that SA and dnOPDA play roles in resistance against fungal pathogens, which are presumably necrotrophs, in M. polymorpha.

\section{Results}

Isolation and morphological characteristics of the MI filamentous microbes

In order to identify the natural pathogens of M. polymorpha, plants showing disease-like symptoms were collected, and then filamentous microbes were isolated from the diseased tissues. As a result, we isolated four distinct filamentous microbes and termed them MI1-4 based on the morphology of colonies (Fig. 1A-D). On potato dextrose agar (PDA), MI1, MI3 and MI4 formed white aerial mycelia and filled 9-cm-diameter plates after $6 \mathrm{~d}$ of incubation at $26^{\circ} \mathrm{C}$ in the dark (Fig. $1 \mathrm{~A}, \mathrm{C}$, D). Scanning electron microscopy (SEM) images of the isolates on PDA are shown in Supplementary Fig. S1. Aerial mycelia of $\mathrm{MI3}$ and MI4 were vigorous with a dense mass of hyphae. However, the aerial mycelium of MI4 was less homogeneous compared to MI3 (Fig. 1C, D). MI2 formed a milky heterogeneous aerial mycelium and its hyphal growth was slower than the other isolates (Fig. 1B). The morphological features of these four isolates were not altered when they were grown under white fluorescent light. After 2 months of culture on PDA, formation of fruiting body-like structures was observed for MI1, MI2 and MI3 (Fig. 1E-G), although none of the four isolates produced spores under our tested growth conditions.

\section{The fungal isolates belong to the order Polyporales}

For molecular identification of the isolates, genomic DNA was extracted from liquid cultures and the rDNA-ITS region was
PCR-amplified and sequenced. Phylogenetic analysis of the rDNA ITS1-5.8S-ITS2 regions revealed that all four fungal isolates belong to Irpicaceae or Phanerochaetaceae families in the Polyporales order (Fig. 1H). The sequenced rDNA-ITS region of MI1 was $100 \%$ identical to I. lacteus; MI2 was $99 \%$ identical to Phaeophlebiopsis peniphoroides; and MI3 and MI4 were 99\% identical to B. adusta. Therefore, we named the isolates I. lacteus MI1, P. peniphoroide MI2, B. adusta MI3 and B. adusta MI4 (Fig. 1H). Many species within the Polyporales are saprotrophic, and Irpicaceae and Phanerochaetaceae fungi are known to produce a white rot by degrading lignin, suggesting that these fungal isolates are necrotrophic pathogens of $M$. polymorpha.

\section{Pathogenicity of the isolated fungi for M. polymorpha}

In order to establish new pathosystems to study the immune system of $M$. polymorpha, we tested whether the isolated fungi can infect and cause disease on M. polymorpha growing axenically in vitro on Gamborg's B5 agar plates. We grew the fungi in liquid potato dextrose broth medium, fragmented them using a homogenizer and adjusted the $\mathrm{OD}_{600}$ for inoculation because we were not able to obtain spores from these fungi. Fourteen-day-old thalli of M. polymorpha Tak-1 (male) and M. polymorpha Tak-2 (female) were challenged with two different concentrations of fungi (Fig. 2A). The Marchantia thalli inoculated with I. lacteus MI1, B. adusta MI3 and B. adusta MI4 exhibited macroscopic disease symptoms, which developed in a concentration-dependent manner (Fig. 2C and Supplementary Figs. S2-S4). Among these three fungi, I. lacteus MI1 exhibited the strongest pathogenicity for $M$. polymorpha and had completely killed the Marchantia thalli by 5 to $6 \mathrm{~d}$ postinoculation (dpi) (Fig. 2C). Although B. adusta MI3 and B. adusta MI4 are closely related (Fig. $1 \mathrm{H}$ ), the pathogenicity of $B$. adusta MI4 was slightly higher compared to B. adusta $\mathrm{MI} 3$ (Fig. 2C). In contrast, we did not observe any obvious P. peniophoroides MI2 infection on Marchantia thalli under these experimental conditions up to $11 \mathrm{dpi}$ (Fig. 2C). It should be noted that observed differences between Tak- 1 and Tak- 2 strains for their susceptibility to the fungi could be due to their differential growth or development of thalli. Based on these observations, we classified the pathogenicity of these fungi on M. polymorpha as shown in Table 1. We decided to use I. lacteus MI1 for further analyses because of its robust pathogenicity for the Marchantia thalli in our experimental system.

\section{SA and/or OPDA treatments alter the susceptibility of M. polymorpha to I. lacteus MI 1 infection}

Irpex lacteus is one of the most common wood-decaying necrotrophs, and, therefore, the I. lacteus MI1-M. polymorpha pathosystem could be used as a model for studying necrotrophic fungus-liverwort interactions. In seed plants, the JA pathway is primarily required for resistance against necrotrophic pathogens and herbivorous insects, and its activity is often suppressed by the SA pathway as in Arabidopsis. In M. polymorpha, dn-OPDA rather than JA-lle functions as a phytohormone, which binds to the MpCOI1 receptor. Mpcoi1 mutants exhibit enhanced susceptibility to the generalist herbivore $S$. littoralis (Monte et al. 2018), suggesting a possible role of $d n$-OPDA in 


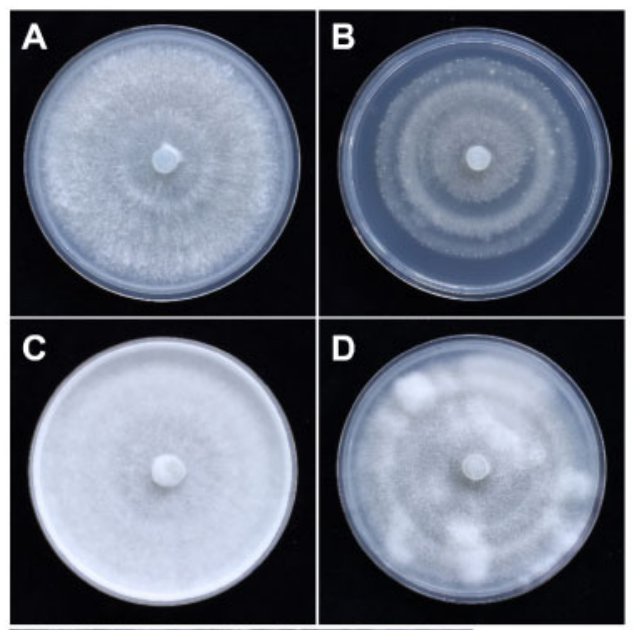

H
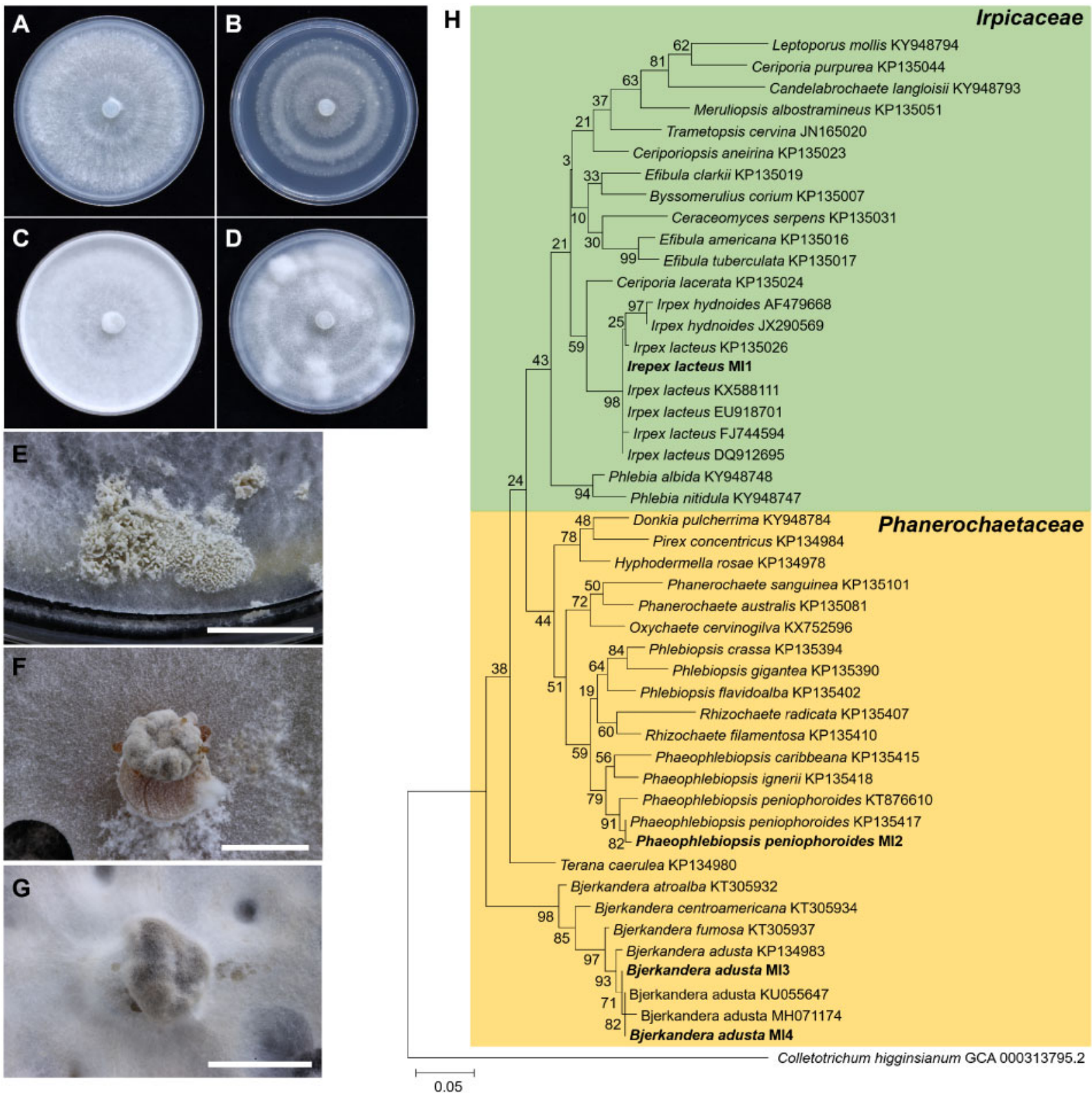

Fig. 1 Fungal strains isolated from diseased Marchantia polymorpha Tak-1. (A-D) Colonies of Irpex lacteus MI1 (A), Phaeophlebiopsis peniophoroides MI2 (B), Bjerkandera adusta MI3 (C) and Bjerkandera adusta MI4 (D) grown on PDA media in a 9-cm Petri dish for 6 days at $26^{\circ} \mathrm{C}$ in the dark. (E-G) Fruiting bodies of I. lacteus MI1 (E), P. peniophoroides MI2 (F) and B. adusta MI3 (G) grown for 2 months at $26^{\circ} \mathrm{C}$ in the dark. Scale bars $=1 \mathrm{~cm}$. $(\mathrm{H})$ Maximum likelihood tree of fungal strains from Phanerochaetaceae and Irpicaceae families based on the ITS1-5.8S-ITS2 region. MI1, MI2, MI3 and MI4 are isolates from diseased M. polymorpha Tak-1. The sequence of Colletotrichum higginsianum was used as an outgroup. Numbers on the branches represent bootstrap values obtained from 1,000 replications.

resistance against necrotrophic pathogens as well. A number of bryophytes including liverworts have been shown to accumulate SA (Drábková et al. 2015), and we detected SA in M. polymorpha Tak-1 (Supplementary Table S1). However, the contribution of SA to M. polymorpha immunity and possible crosstalk between SA and dn-OPDA pathways has not yet been clarified. To address these questions, we tested whether exogenous applications of SA and/or OPDA, the dn-OPDA precursor, to M. polymorpha could influence I. lacteus MI1 infection.

Thalli of 14-day-old $M$. polymorpha Tak-1 were soaked in water (mock), $500 \mu \mathrm{M} \mathrm{SA}, 1,000 \mu \mathrm{M}$ SA or $50 \mu \mathrm{M}$ OPDA for $3 \mathrm{~h}$ and then inoculated with I. lacteus MI1 (Fig. 3A). Macroscopic disease symptoms appeared at $5 \mathrm{dpi}$, and the SA treatments resulted in a significant promotion of lesion development at 6 and $7 \mathrm{dpi}$ compared to the mock treatment (Fig. 3B, C and Supplementary Fig. S5). In contrast, the OPDA treatment showed no effect on the lesion development, which is reminiscent of the inability of exogenous JA treatments to protect wildtype Arabidopsis plants against certain necrotrophic pathogens (Thomma et al. 1998). The SA or OPDA treatments did not affect plant health (Supplementary Fig. S5), and we confirmed that SA does not promote but rather suppresses I. lacteus MI1 
A

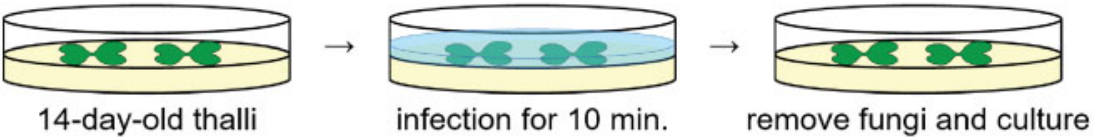

B

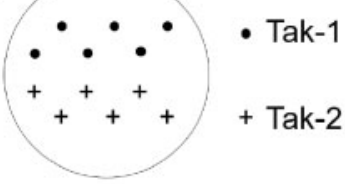

B. adusta MI4

\subsection{2} 0.02 0.002
0.0202

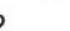

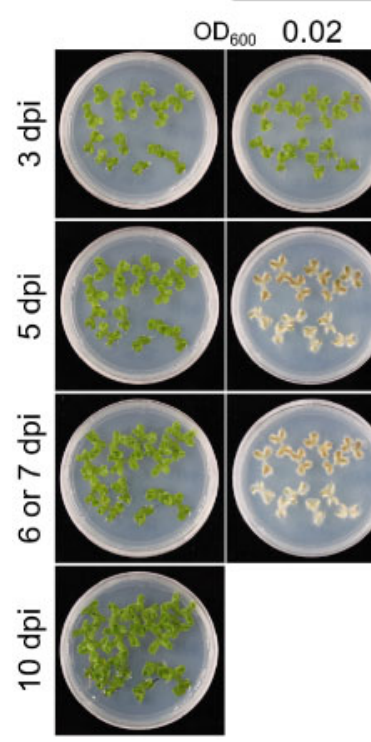
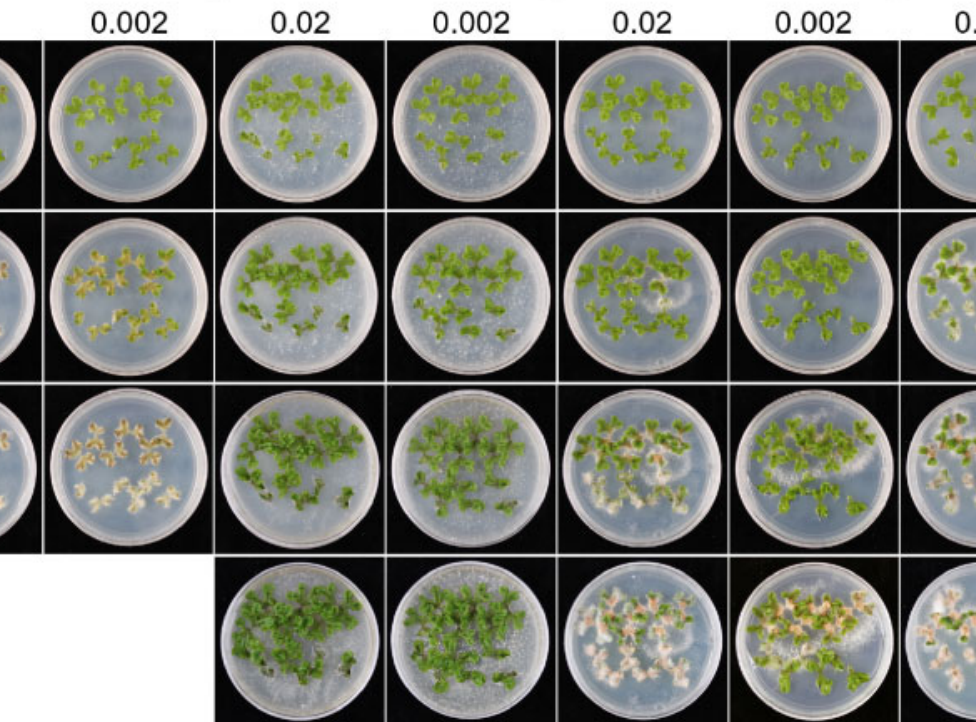

Fig. 2 Pathogenicity of the fungal isolates for Marchantia polymorpha. (A) Schematic presentation of fungal inoculations of M. polymorpha. (B) Arrangement of M. polymorpha Tak-1 and Tak-2 on plates. (C) Inoculation of M. polymorpha with the fungal isolates. For I. lacteus MI1 infection, the image was taken at 6 days postinoculation (dpi). For the rest, images are from $7 \mathrm{dpi}$.

Table 1 Fungal strains isolated in this study

\begin{tabular}{lclc}
\hline Species & Isolate & Isolation locality & $\begin{array}{c}\text { Pathogenicity to } \\
\text { M. polymorpha }\end{array}$ \\
\hline $\begin{array}{l}\text { Irpex lacteus } \\
\begin{array}{l}\text { Phanerochaete } \\
\text { peniophoroides }\end{array}\end{array}$ & MI1 & Kitashirakawa, Kyoto & ++ \\
$\begin{array}{l}\text { Bjerkandera adusta } \\
\text { Bjerkandera adusta }\end{array}$ & MI3 & Kitashirakawa, Kyoto & \pm \\
\hline
\end{tabular}

${ }^{\text {a }}$ Pathogenicity under the experimental conditions used in this study.

growth on Gamborg's B5 agar (Supplementary Fig. S6). These results indicate that, as in many seed plants, activation of the SA pathway compromises resistance against necrotrophic pathogens in M. polymorpha. This suggests the existence of antagonistic crosstalk between the SA and dn-OPDA pathways in M. polymorpha.

As the possible effects of a single OPDA treatment can be very transient, we next added $500 \mu \mathrm{M} \mathrm{SA}$ and/or $1 \mu \mathrm{M} \mathrm{dn}$-cisOPDA to the Gamborg's B5 agar plates, thus continuously exposing the Marchantia thalli to these phytohormones during infection (Fig. 4A). The stimulatory effect of SA on I. lacteus MI1 infection was confirmed under these conditions, but sustained application of dn-cis-OPDA did not affect the lesion development as we previously observed (Fig. 4B, C and Supplementary Figs. S7, S8). Intriguingly, however, the ability of SA to promote I. lacteus MI1 infection was compromised by co-treatment with
dn-cis-OPDA (Fig. 4B, C and Supplementary Figs. S7, S8). These results suggest that $d n$-cis-OPDA suppresses the SA pathway and thereby contributes to resistance against necrotrophic pathogens in M. polymorpha.

To further verify the involvement of the OPDA pathway in resistance against necrotrophic pathogens, we challenged the Mpcoi1-2 mutant allele (male) (Monte et al. 2018) with I. lacteus MI1. The Mpcoi1-2 mutant displayed enhanced susceptibility against I. lacteus MI1 compared to $M$. polymorpha Tak-1 (Fig. 5B, C and Supplementary Figs. S9, S10), which suggests that the OPDA pathway positively regulates resistance against necrotrophic pathogens in M. polymorpha.

\section{Discussion}

To date, there are hardly any reports that describe pathogenic microbes that naturally infect and cause disease in M. polymorpha, which hinders the use of M. polymorpha in EvoMPMI studies (Carella and Schornack 2018). The recent finding that the hemi-biotrophic oomycete pathogen P. palmivora can colonize M. polymorpha enabled the study of liverwort-pathogen interactions at the molecular level (Carella et al. 2018). Nevertheless, to enable the study of a range of different interactions, other types of $M$. polymorpha-infecting pathogens are needed. In this work, we have isolated four fungal strains, which are most likely necrotrophic pathogens, from diseased M. polymorpha, and demonstrated that the I. lacteus MI 1 isolate can be 
A

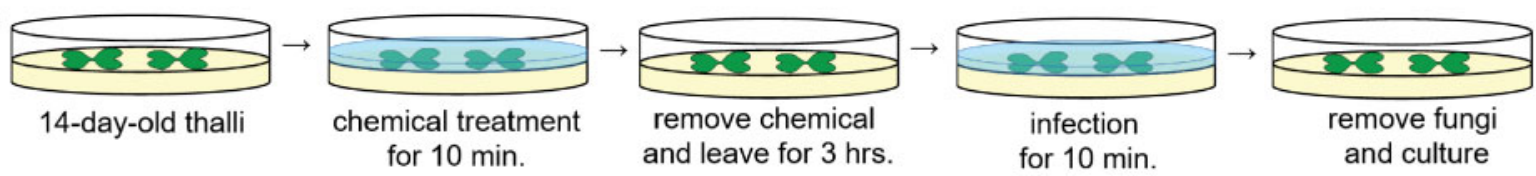

B

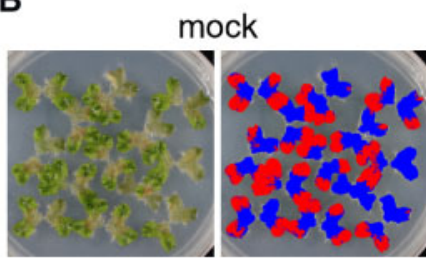

$1000 \mu \mathrm{M} \mathrm{SA}$

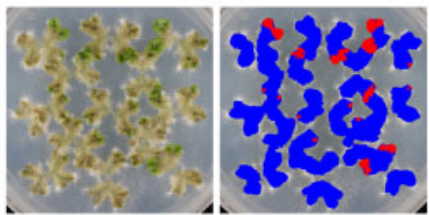

$500 \mu \mathrm{M}$ SA

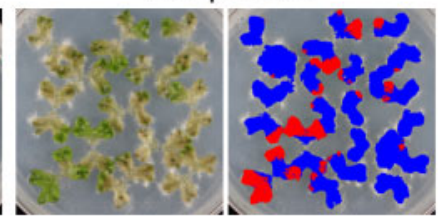

$50 \mu \mathrm{M}$ OPDA
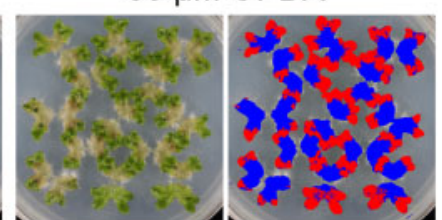

C

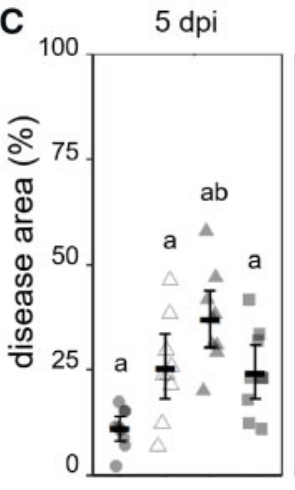

- mock

$\triangle 500 \mu \mathrm{M} \mathrm{SA}$
$6 \mathrm{dpi}$

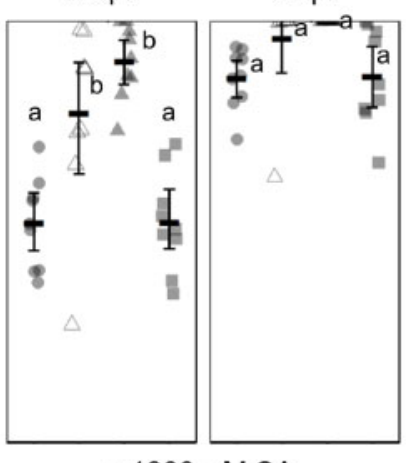

$\triangle 1000 \mu \mathrm{M} \mathrm{SA}$

= $50 \mu \mathrm{M}$ OPDA

Fig. 3 SA treatment promotes Irpex lacteus MI1 infection of Marchantia polymorpha Tak-1. (A) Schematic representation of chemical treatment and subsequent fungal inoculation. (B) Images of M. polymorpha Tak-1 thalli infected with I. lacteus MI1. Treatment conditions are indicated on the top of the panels. Images at $6 \mathrm{dpi}$ (left) and their healthy and lesion-bearing areas detected by the ImageJ-based PIDIQ method (right) are shown. Healthy and lesion-bearing areas are colored in red and blue, respectively. (C) Effects of SA or OPDA on M. polymorpha Tak-1 infection with I. lacteus MI1. The proportions of the lesion-bearing areas at 5-7 dpi are shown. Gray circles, white triangles, gray triangles and gray squares represent individual data points for each condition indicated. Within each group, the middle horizontal bar is the mean, and the error bars reflect $95 \%$ confidence intervals. Different letters indicate significant differences between treatments at each dpi ( $n=9, P<0.01$, Tukey's HSD test).

A

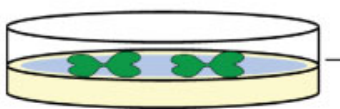

14-day-old thalli grown on solid medium covered with cellophane disc

B

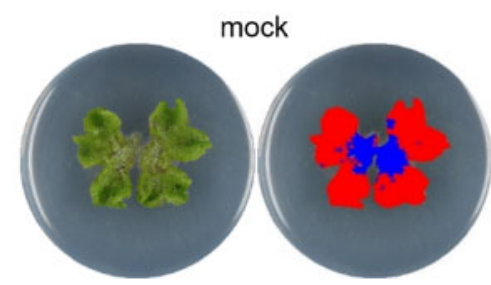

$1 \mu \mathrm{M}$ dn-cis-OPDA
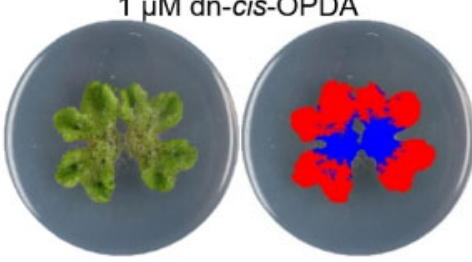

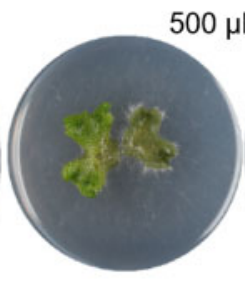

$500 \mu \mathrm{M} \mathrm{SA}+1 \mu \mathrm{M}$ dn-cis-OPDA

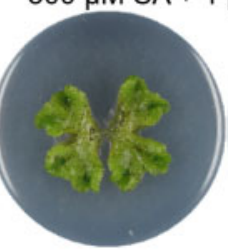

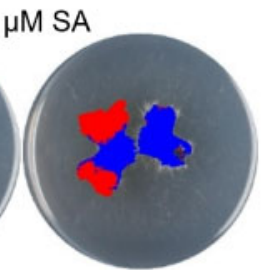

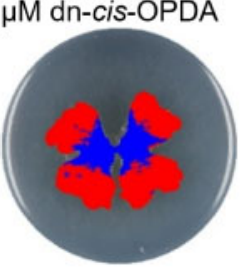

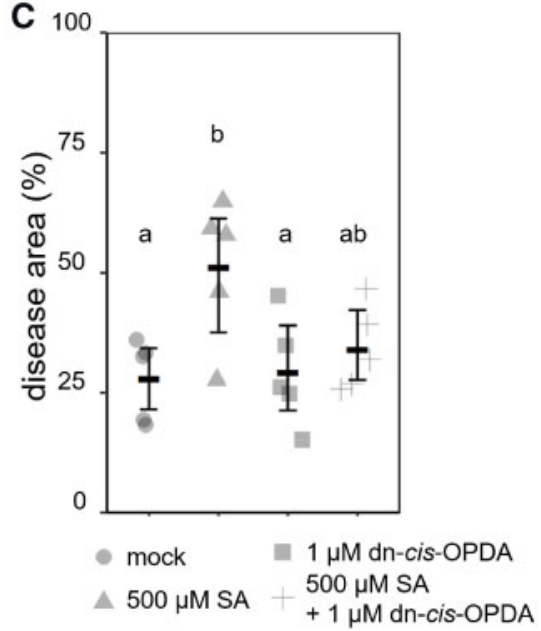

Fig. 4 SA effect is suppressed by dn-cis-OPDA co-treatment. (A) Schematic representation of chemical treatment and fungal inoculation. (B) Images of M. polymorpha Tak-1 thalli infected with I. lacteus MI1. Treatment conditions are indicated on the top of the panels. Representative images at $4 \mathrm{dpi}$ (left) and their healthy and lesion-bearing areas detected by the ImageJ-based PIDIQ method (right) are shown. Healthy and lesion-bearing areas are colored in red and blue, respectively. (C) Effects of SA or dn-cis-OPDA on M. polymorpha Tak-1 infection with I. lacteus MI1. The proportions of the lesion-bearing areas at $4 \mathrm{dpi}$ are shown. Circles, triangles, squares and plus signs represent individual data points for each condition indicated. Within each group, the middle horizontal bar is the mean, and the error bars reflect $95 \%$ confidence intervals. Different letters indicate significant differences between treatments at each dpi $(n=5, P<0.05$, Tukey's HSD test). 
A
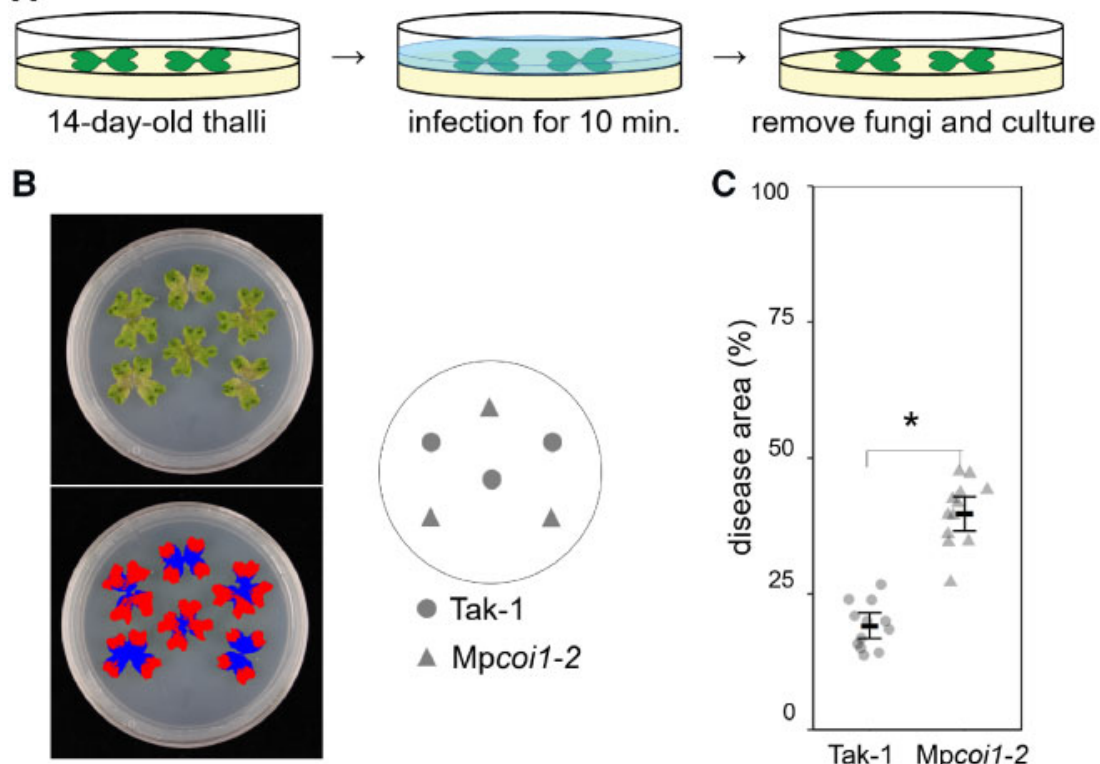

Fig. 5 Mpcoi1 mutant is susceptible to Irpex lacteus MI1. (A) Schematic presentation of fungal inoculations of M. polymorpha. (B) Images of M. polymorpha Tak-1 and Mpcoi1-2 thalli infected with I. lacteus MI1. Representative images at $6 \mathrm{dpi}$ (upper left) with healthy and lesion-bearing areas detected by the ImageJ-based PIDIQ method (lower left) are shown. Healthy and lesion-bearing areas are colored in red and blue, respectively. Arrangement of M. polymorpha Tak-1 and Mpcoi1-2 on the plate (right). (C) Infection of M. polymorpha Tak-1 and Mpcoi1 with I. lacteus MI1. The proportions of the lesion-bearing areas at $6 \mathrm{dpi}$ are shown. Circles and triangles represent individual data points for each genotype indicated. Within each group, the middle horizontal bar is the mean, and the error bars reflect $95 \%$ confidence intervals. Asterisks indicate significant differences between M. polymorpha Tak-1 and Mpcoi1-2 ( $n=12, P<0.01$, Student's $t$-test).

used as a model to understand interactions between necrotrophic fungi and liverworts. We anticipate that further bacterial pathogens or biotrophic pathogens of M. polymorpha will be identified in the future.

The white rot fungus I. lacteus is a cosmopolitan species, which produces hydrolases including lignin-degrading enzymes that decompose plant cell walls facilitating its colonization (Novotný et al. 2009). Because of its biotechnological potential, I. lacteus has been studied for many years, and the genomes of the I. lacteus strains F17 and CD2 have been sequenced (Yao et al. 2017, Qin et al. 2018). Interestingly, I. lacteus was previously isolated from the moss $R$. japonicum growing in a field in Japan and was shown to cause disease in P. patens (Lehtonen et al. 2009). Therefore, I. lacteus might be pathogenic for a wide range of bryophytes, and could therefore be generally used as a model necrotroph to study interactions between bryophytes and fungi. In P. patens, the class III peroxidase Prx34 is secreted upon chitosan treatment, and prx34 knock-out mutants displayed susceptibility to the I. lacteus isolate (Lehtonen et al. 2009). Determining the roles of the class III peroxidases during I. lacteus infection in M. polymorpha would contribute to our understanding of the evolution of immune mechanisms within bryophytes.

When we inoculated the I. lacteus MI1, B. adusta MI3 or B. adusta MI4 isolates to M. polymorpha Tak-1 thalli using our method, we could hardly observe fungi growing on the surface of Marchantia thalli. Interestingly, we found that hyphae of the fungi are coming out from air chambers (Supplementary Figs. S2E, F, S3B, D), which suggests that the fungal isolates enter air chambers for their successful growth and infection. These observations are very interesting because the hemibiotrophic oomycete pathogen $P$. palmivora was shown to take similar approach to infect $M$. polymorpha (Carella et al. 2018). These findings suggest that air chambers are crucial battlefields between the host and invading microbes.

JA plays a pivotal role in defense responses against necrotrophic pathogens in seed plants. In M. polymorpha, OPDA is expected to play a similar role as JA in seed plants (Monte et al. 2018). However, OPDA treatment of $M$. polymorpha failed to boost resistance against the I. lacteus MI1 isolate under our experimental conditions. This observation is consistent with the fact that JA treatment can boost resistance against the necrotrophic fungus Alternaria brassicicola only in pad3 mutants but not in wild-type Col-0 plants in Arabidopsis (Thomma et al. 1998, Spoel et al. 2007). In Arabidopsis, the JA-insensitive coi1 mutants also display susceptibility to A. brassicicola, and, therefore, either JA-dependent defense activation or PAD3-dependent camalexin synthesis seems to be sufficient for the resistance against necrotrophic pathogens. Accordingly, the increased susceptibility of the OPDA-insensitive Mpcoi mutant to I. lacteus MI1 (Monte et al. 2018) indicates that OPDAdependent defense activation plays a role in resistance against necrotrophic fungi in M. polymorpha.

SA levels in 3-week-old thalli of M. polymorpha Tak-1, which were grown on Gamborg's B5 agar plates, were relatively low compared to non-stressed Arabidopsis but were comparable to other analyzed liverworts (Drábková et al. 2015). Interestingly, 6-day-old gemmae of M. polymorpha Tak-1 that were grown in 
Gamborg's B5 liquid media with shaking accumulated more SA compared to the thalli on the plates (Supplementary Table S1). SA levels may differ in different developmental stages. It is also possible that growth in liquid media, a rather stressful condition for gemmae, may have induced SA accumulation. It would be interesting to determine whether biotic stresses affect SA levels in M. polymorpha as in seed plants. We could detect only very low levels of SA glucoside (SAG) in the samples we analyzed (Supplementary Table S1), and we expect that further investigations into SA biosynthesis and metabolism in M. polymorpha will improve our understanding of the physiological roles of SA in liverworts.

The striking observation that SA treatment promoted I. lacteus MI1 infection implies the existence of SA-OPDA crosstalk in $M$. polymorpha, which is similar to the antagonistic SA-JA crosstalk in seed plants. In line with this observation, OPDA co-treatment resulted in suppression of the SA-dependent stimulatory effect. This suggests that OPDA is prioritized over SA in M. polymorpha, which is opposite to the case in Arabidopsis (Spoel et al. 2003, Spoel et al. 2007, Leon-Reyes et al. 2010). Further molecular studies are needed to draw solid conclusions regarding the SA-OPDA crosstalk in M. polymorpha. For instance, generation and use of a transgenic M. polymorpha that expresses NahG (Gaffney et al. 1993), which degrades endogenous SA to catechol may give us more insight into this crosstalk. Considering the importance of SA-JA antagonism in seed plants in dealing with simultaneous or consecutive attack by multiple contrasting pathogens, we speculate that SA treatment might boost the resistance of $M$. polymorpha against biotrophic and hemi-biotrophic pathogens. Intriguingly, it was shown that SA treatment could induce resistance against the necrotrophic bacterial pathogen E. carotovora in $P$. patens (Andersson et al. 2005). Therefore, the roles of SA in defense responses may differ between mosses and liverworts.

\section{Materials and Methods}

\section{Plant material and growth condition}

Marchantia polymorpha, accession Takaragaike-1 (Tak-1; male) and Takaragaike-2 (Tak-2; female) were used for experiments. M. polymorpha was cultured at $22^{\circ} \mathrm{C}$ on half-strength Gamborg's B5 medium (Gamborg et al. 1968) containing $1 \%$ agar under $50-60 \mu \mathrm{mol}$ photons $\mathrm{m}^{-2} \mathrm{~s}^{-1}$ continuous white LED (light-emitting diode) light.

\section{Fungal isolation}

In vitro grown thalli of M. polymorpha Tak-1 were exposed to outside air for a while at Kitashirakawa, Kyoto, and then further cultivated in a growth chamber at $22^{\circ} \mathrm{C}$ under continuous white light. For isolation of natural pathogens, $3 \mathrm{~mm}^{2}$ pieces were cut from $M$. polymorpha Tak-1 with disease-like symptoms collected at Kyoto, Japan. Small pieces were soaked in $70 \%(\mathrm{w} / \mathrm{v})$ ethanol for $1 \mathrm{~min}$, and dipped into $1 \%(\mathrm{w} / \mathrm{v})$ sodium hypochlorite solution for $3 \mathrm{~min}$. Pieces were then immediately rinsed two times with sterilized water, placed on $1 \%$ water agar plates containing $0.01 \%(w / v)$ streptomycin sulfate and incubated at $26^{\circ} \mathrm{C}$ for a few days. After confirming the growth of fungal colonies, a hyphal tip from each developing colony was transferred to a PDA (BD, Franklin Lakes, NJ, USA) plate.

\section{DNA amplification and extraction}

Isolates were cultured on PDA plates at $26^{\circ} \mathrm{C}$ for $7 \mathrm{~d}$. Ten pieces of mycelial plug ( $1 \mathrm{~mm}$ diameter) of $\mathrm{MI} 1, \mathrm{MI} 3$ and $\mathrm{MI} 4$ were transferred to $300 \mathrm{ml}$ flasks containing $100 \mathrm{ml}$ of $\mathrm{CM}$ liquid medium consisting of $0.3 \%(\mathrm{w} / \mathrm{v})$ casamino acids, $0.3 \%(\mathrm{w} / \mathrm{v})$ yeast extract and $0.5 \%(\mathrm{w} / \mathrm{v})$ sucrose. Ten pieces of mycelial plug of the MI2 isolate were transferred to $300 \mathrm{ml}$ flasks containing $100 \mathrm{ml}$ of liquid medium consisting of $0.3 \%(\mathrm{w} / \mathrm{v})$ yeast extract and $0.5 \%(\mathrm{w} / \mathrm{v})$ sucrose. Flasks were shaken at $90 \mathrm{rpm}$ at $26^{\circ} \mathrm{C}$ for 2-4 d. Mycelia produced in liquid media were filtered, freeze-dried, frozen with liquid nitrogen and ground to powder using a mortar and pestle. Genomic DNA was extracted using DNeasy Plant Kits (QIAGEN, Hilden, Germany), according to the manufacturer's protocol.

\section{PCR amplification}

PCR was performed using $5 \mathrm{ng}$ of fungal genomic DNA. ITS1F ( $5^{\prime}$-GTAAC AAGGTYTCCGT- $3^{\prime}$ ) and ITS4 (5'-TCCTCCGCTTATTGATATG- $3^{\prime}$ ) (White et al. 1990, Gardes and Bruns 1993) were used for the amplification of the nucleotide sequences of rDNA ITS1-5.8S-ITS2 regions. The PCR-amplified products were purified using Wizard ${ }^{\circledR}$ SV Gels and a PCR Clean-Up System (Promega, Madison, WI, USA) and then sequenced.

\section{Sequence alignment and phylogenetic analysis}

Multiple alignment of nucleotide sequences was performed using MUSCLE. Phylogenetic analysis was performed using PhyML (http://www.atgc-montpel lier.fr/phyml/) with 1,000 bootstrap replications. For multiple genetic genealogy analysis, nucleotide sequences of rDNA ITS1-5.8S-ITS2 regions from Polyporales were downloaded from GenBank (https://www.ncbi.nlm.nih.gov).

\section{Inoculation test}

Marchantia gemmae were placed on half-strength Gamborg's B5 agar plates and grown for $14 \mathrm{~d}$ under continuous white LED light or a $14 \mathrm{~h}$ light/10 $\mathrm{h}$ dark cycle. For preparation of inoculum, mycelium was cultured in CM liquid medium at $21^{\circ} \mathrm{C}$ for $7 \mathrm{~d}$ on shakers running at $120 \mathrm{rpm}$. About $1 \mathrm{~cm}$ of mycelium ball was rinsed with sterilized water, homogenized with ULTRA-TURRAX T8 (IKA Labortechnik, Staufen, Germany) and suspended into $5 \mathrm{ml}$ sterilized water. Mycelium suspension at an $\mathrm{OD}_{600}$ of 0.02 or 0.002 was decanted into the Marchantia culture plate and incubated for $10 \mathrm{~min}$. The mycelium suspension was removed, and then Marchantia thalli were further cultured for the indicated number of days.

For SA or OPDA treatments in Fig. 3, sodium salicylate (Sigma-Aldrich, Munich, Germany) and OPDA (Cayman Chemical, Ann Arbor, MI, USA) were dissolved in $1.47 \%$ ethanol, and $1.47 \%$ ethanol was used for the mock treatment. The chemical solutions were added to the Marchantia culture plates and the 14day-old thalli were immersed in the chemicals for $10 \mathrm{~min}$. After removal of the chemical solutions, treated thalli were incubated for $3 \mathrm{~h}$, and then the mycelium suspension at an $\mathrm{OD}_{600}$ of 0.002 was inoculated as described above.

For SA and/or dn-cis-OPDA application to the growth media in Fig. 4, Marchantia gemmae were grown on half-strength Gamborg's B5 agar covered with a cellophane disc (Diagonal, Münster, Germany) to avoid rhizoids growing into the media for easy transfer of thalli. SA and dn-OPDA (Cayman Chemical, Ann Arbor, MI, USA) were dissolved in $0.026 \%$ ethanol, and $0.026 \%$ ethanol was used as the mock control. Fourteen-day-old thalli were dipped in the chemical solutions for $3 \mathrm{~h}$ followed by incubation with the mycelium suspension at an $\mathrm{OD}_{600}$ of 0.002 for $10 \mathrm{~min}$. Then, treated thalli were cultured on half-strength Gamborg's B5 agar plates supplemented with the chemicals.

\section{Measurement of disease area}

To measure healthy and lesion-bearing areas of M. polymorpha infected with I. lacteus MI1, Imagej-based macro plant immunity and disease image-based quantification (PIDIQ) was used (Laflamme et al. 2016). The 'Threshold Color' value was adjusted as described in the supplemental DATA (Supplementary ImageJ macro 1-3). Statistical analysis was performed using Tukey's HSD test with R software version 3.2.3 (https://www.r-project.org/).

\section{Quantification of SA}

The quantification of SA and SAG was performed as described previously (Seo et al. 1995, Betsuyaku et al. 2018). Briefly, three-week-old thalli of M. polymorpha Tak-1 that were grown on Gamborg's B5 agar plates or 6-day-old gemmae of $M$. polymorpha Tak-1 that were grown in Gamborg's B5 liquid media with shaking 
were frozen and ground using liquid nitrogen. SA and SAG were extracted with $90 \%$ methanol, and SAG was converted to SA by $\beta$-glucosidase treatment. After separation by HPLC (Shimadzu) with an ODS column ( $\mu$-Bondasphere C18, $150 \mathrm{~mm} \times$ ID3.9 mm, $5 \mu \mathrm{m}, 100 \mathrm{~A}$; Waters), SA levels were determined using a fluorescence detector (RF-20A; Shimadzu) with an excitation wavelength of $313 \mathrm{~nm}$ and an emission wavelength of $405 \mathrm{~nm}$.

\section{Cryo-SEM}

Samples were mounted on copper-made sample holders, shock frozen in liquid nitrogen and sublimated, sputtered with Gold/Palladium mixture ( $80 \%$ Gold/ 20\% Palladium) using an Emitech K1250X cryo system, and then images were taken using a Zeiss Supra 40VP scanning electron microscope (Carl Zeiss NTS).

\section{Supplementary Data}

Supplementary data are available at PCP online.

\section{Funding}

The Max-Planck-Gesellschaft to H.N.; Japan Society for the Promotion of Science KAKENHI [17K07665 to S.K.]; and Spanish Ministry for Science and Innovation grant [BIO201677216-R (MINECO/FEDER) to R.S.].

\section{Acknowledgments}

We thank Yuko Nomura (RIKEN), Shouko Yamazaki (RIKEN) and Takeshi Hosaka (Shinshu University) for technical assistance. We also thank Selena Gimenez-Ibanez (CNB-CSIC) for suggestions to improve the manuscript. We also thank Neysan Donnelly for editing the manuscript.

\section{Disclosures}

The authors have no conflicts of interest to declare.

\section{References}

Andersson, R.A., Akita, M., Pirhonen, M., Gammelgård, E. and Valkonen, J.P. T. (2005) Moss-Erwinia pathosystem reveals possible similarities in pathogenesis and pathogen defense in vascular and nonvascular plants. J. Gen. Plant Pathol. 71: 23-28.

Berens, M.L., Berry, H.M., Mine, A., Argueso, C.T. and Tsuda, K. (2017) Evolution of hormone signaling networks in plant defense. Annu. Rev. Phytopathol. 55: 401-425.

Berger, F., Bowman, J.L. and Kohchi, T. (2016) Marchantia. Curr. Biol. 26: R186-R187.

Betsuyaku, S., Katou, S., Takebayashi, Y., Sakakibara, H., Nomura, N. and Fukuda, H. (2018) Salicylic acid and jasmonic acid pathways are activated in spatially different domains around the infection site during effector-triggered immunity in Arabidopsis thaliana. Plant Cell Physiol. 59: 8-16.

Bidartondo, M.I. and Duckett, J.G. (2010) Conservative ecological and evolutionary patterns in liverwort-fungal symbioses. Proc. $R$. Soc. $B$ 277: 485-492.

Bonfante, P. and Genre, A. (2010) Mechanisms underlying beneficial plant-fungus interactions in mycorrhizal symbiosis. Nat. Commun. 1: 1-11.

Bowman, J.L., Kohchi, T., Yamato, K.T., Jenkins, J., Shu, S., Ishizaki, K., et al. (2017) Insights into land plant evolution garnered from the Marchantia polymorpha genome. Cell 171: 287-304.e15.
Bressendorff, S., Azevedo, R., Kenchappa, C.S., Ponce de León, I., Olsen, J.V., Rasmussen, M.W., et al. (2016) An innate immunity pathway in the moss Physcomitrella patens. Plant Cell 28: 1328-1342.

Carella, P., Gogleva, A., Tomaselli, M., Alfs, C. and Schornack, S. (2018) Phytophthora palmivora establishes tissue-specific intracellular infection structures in the earliest divergent land plant lineage. Proc. Natl. Acad. Sci. USA 115: E3846-E3855.

Carella, P. and Schornack, S. (2018) Manipulation of bryophyte hosts by pathogenic and symbiotic microbes. Plant Cell Physiol. 59: 656-665.

Castro, A., Vidal, S. and Ponce de León, I. (2016) Moss pathogenesis-related-10 protein enhances resistance to Pythium irregulare in Physcomitrella patens and Arabidopsis thaliana. Front. Plant Sci. 7: 580.

Davey, M.L., Tsuneda, A. and Currah, R.S. (2009) Pathogenesis of bryophyte hosts by the ascomycete Atradidymella muscivora. Am. J. Bot. 96: $1274-1280$.

Delwiche, C.F. and Cooper, E.D. (2015) The evolutionary origin of a terrestrial flora. Curr. Biol. 25: R899-R910.

Delwiche, C.F., Goodman, C.A. and Chang, C. (2017) Land plant model systems branch out. Cell 171: 265-266.

Delwiche, C.F. and Timme, R.E. (2011) Plants. Curr. Biol. 21: 417-422.

Drábková, L.Z., Dobrev, P.I. and Motyka, V. (2015) Phytohormone profiling across the bryophytes. PLoS One 10: e0125411.

Duckett, J.G., Ligrone, R., Renzaglia, K.S. and Pressel, S. (2014) Pegged and smooth rhizoids in complex thalloid liverworts (Marchantiopsida): structure, function and evolution: dimorphic rhizoids in Marchantiopsida. Bot. J. Linn. Soc. 174: 68-92.

Eklund, D.M., Ishizaki, K., Flores-Sandoval, E., Kikuchi, S., Takebayashi, Y. and Tsukamoto, S. (2015) Auxin produced by the indole-3-pyruvic acid pathway regulates development and gemmae dormancy in the liverwort Marchantia polymorpha. Plant Cell 27: 1650-1669.

Field, K.J. and Pressel, S. (2018) Unity in diversity: structural and functional insights into the ancient partnerships between plants and fungi. New Phytol. 220: 996-1011.

Field, K.J., Pressel, S., Duckett, J.G., Rimington, W.R. and Bidartondo, M.I. (2015) Symbiotic options for the conquest of land. Trends Ecol. Evol. 30: 477-486.

Field, K.J., Rimington, W.R., Bidartondo, M.I., Allinson, K.E., Beerling, D.J., Cameron, D.D., et al. (2016) Functional analysis of liverworts in dual symbiosis with Glomeromycota and Mucoromycotina fungi under a simulated Palaeozoic CO2 decline. ISME J. 10: 1514-1526.

Flores-Sandoval, E., Dierschke, T., Fisher, T.J. and Bowman, J.L. (2016) Efficient and inducible use of artificial microRNAs in Marchantia polymorpha. Plant Cell Physiol. 57: 281-290.

Gaffney, T., Friedrich, L., Vernooij, B., Negrotto, D., Nye, G., Uknes, S., et al. (1993) Requirement of salicylic acid for the induction of systemic acquired resistance. Science 261: 754-756.

Gamborg, O.L., Miller, R.A. and Ojima, K. (1968) Nutrient requirements of suspension sultures of soybean root cells. Exp. Cell Res. 50: 151-158.

Gardes, M. and Bruns, T.D. (1993) ITS primers with enhanced specificity for basidiomycetes-application to the identification of mycorrhizae and rusts. Mol. Ecol. 2: 113-118.

Glazebrook, J. (2005) Contrasting mechanisms of defense against biotrophic and necrotrophic pathogens. Annu. Rev. Phytopathol. 43: 205-227.

Harrison, C.J. (2017) Development and genetics in the evolution of land plant body plans. Philos. Trans. R. Soc. B Biol. Sci. 372: 20150490.

Hoysted, G.A., Kowal, J., Jacob, A., Rimington, W.R., Duckett, J.G., Pressel, S., et al. (2018) A mycorrhizal revolution. Curr. Opin. Plant Biol. 44: 1-6.

Humphreys, C.P., Franks, P.J., Rees, M., Bidartondo, M.I., Leake, J.R. and Beerling, D.J. (2010) Mutualistic mycorrhiza-like symbiosis in the most ancient group of land plants. Nat. Commun. 1: 103.

Ishizaki, K., Chiyoda, S., Yamato, K.T. and Kohchi, T. (2008) Agrobacterium-mediated transformation of the haploid liverwort Marchantia polymorpha L., an emerging model for plant biology. Plant Cell Physiol. 49: 1084-1091. 
Ishizaki, K., Johzuka-Hisatomi, Y., Ishida, S., lida, S. and Kohchi, T. (2013) Homologous recombination-mediated gene targeting in the liverwort Marchantia polymorpha L. Sci. Rep. 3: 1532.

Ishizaki, K., Nishihama, R., Ueda, M., Inoue, K., Ishida, S., Nishimura, Y., et al. (2015) Development of gateway binary vector series with four different selection markers for the liverwort Marchantia polymorpha. PLoS One 10: e0138876.

Ishizaki, K., Nishihama, R., Yamato, K.T. and Kohchi, T. (2016) Molecular genetic tools and techniques for Marchantia polymorpha research. Plant Cell Physiol. 57: 262-270.

Jones, V.A.S. and Dolan, L. (2012) The evolution of root hairs and rhizoids. Ann. Bot. 110: 205-212.

Kato, H., Ishizaki, K., Kouno, M., Shirakawa, M., Bowman, J.L., Nishihama, R., et al. (2015) Auxin-mediated transcriptional system with a minimal set of components is critical for morphogenesis through the life cycle in Marchantia polymorpha. PLoS Genet. 11: e1005084.

Koornneef, A. and Pieterse, C.M.J. (2008) Cross talk in defense signaling. Plant Physiol. 146: 839-844.

Kubota, A., Ishizaki, K., Hosaka, M. and Kohchi, T. (2013) Efficient Agrobacterium-mediated transformation of the liverwort Marchantia polymorpha using regenerating thalli. Biosci. Biotechnol. Biochem. 77: 167-172.

Laflamme, B., Middleton, M., Lo, T., Desveaux, D. and Guttman, D.S. (2016) Image-based quantification of plant immunity and disease. Mol. Plant Microbe Interact. 29: 919-924.

Lehtonen, M.T., Akita, M., Kalkkinen, N., Ahola-livarinen, E., Rönnholm, G., Somervuo, P., et al. (2009) Quickly-released peroxidase of moss in defense against fungal invaders. New Phytol. 183: 432-443.

Leon-Reyes, A., Van der Does, D., De Lange, E.S., Delker, C., Wasternack, C., Van Wees, S.C.M., et al. (2010) Salicylate-mediated suppression of jasmonate-responsive gene expression in Arabidopsis is targeted downstream of the jasmonate biosynthesis pathway. Planta 232: 1423-1432.

Ligrone, R., Carafa, A., Lumini, E., Bianciotto, V., Bonfante, P. and Duckett, J.G. (2007) Glomeromycotean associations in liverworts: a molecular, cellular, and taxonomic analysis. Am. J. Bot. 94: 1756-1777.

Lockhart, J. (2015) The elegant simplicity of the liverwort Marchantia polymorpha. Plant Cell 27: 1565-1565.

Martin, F.M., Uroz, S. and Barker, D.G. (2017) Ancestral alliances: plant mutualistic symbioses with fungi and bacteria. Science 356: eaad4501.

Mathieu, S., Cusant, L., Roux, C. and Corradi, N. (2018) Arbuscular mycorrhizal fungi: intraspecific diversity and pangenomes. New Phytol. 220: 1129-1134.

Monte, I., Ishida, S., Zamarreño, A.M., Hamberg, M., Franco-Zorrilla, J.M., García-Casado, G., et al. (2018) Ligand-receptor co-evolution shaped the jasmonate pathway in land plants. Nat. Chem. Biol. 14: 480-488.

Morris, J.L., Puttick, M.N., Clark, J.W., Edwards, D., Kenrick, P., Pressel, S., et al. (2018) The timescale of early land plant evolution. Proc. Natl. Acad. Sci. USA 115: E2274-E2283.

Nelson, J. and Shaw, A.J. (2019) Exploring the natural microbiome of the model liverwort: fungal endophyte diversity in Marchantia polymorpha L. Symbiosis 78: 45-59.

Nelson, J.M., Hauser, D.A., Hinson, R. and Shaw, A.J. (2018) A novel experimental system using the liverwort Marchantia polymorpha and its fungal endophytes reveals diverse and context-dependent effects. New Phytol. 218: 1217-1232.

Nishihama, R., Ishida, S., Urawa, H., Kamei, Y. and Kohchi, T. (2016) Conditional gene expression/deletion systems for Marchantia polymorpha using its own heat-shock promoter and Cre/lox P-mediated site-specific recombination. Plant Cell Physiol. 57: 271-280.

Novotný, Č., Cajthaml, T., Svobodová, K., Šušla, M. and Šašek, V. (2009) Irpex lacteus, a white-rot fungus with biotechnological potential-review. Folia Microbiol. 54: 375-390.

Oliver, J.P., Castro, A., Gaggero, C., Cascón, T., Schmelz, E.A., Castresana, C., et al. (2009) Pythium infection activates conserved plant defense responses in mosses. Planta 230: 569-579.
Overdijk, E.J.R., De Keijzer, J., De Groot, D., Schoina, C., Bouwmeester, K., Ketelaar, T., et al. (2016) Interaction between the moss Physcomitrella patens and Phytophthora: a novel pathosystem for live-cell imaging of subcellular defence. J. Microsc. 263: 171-180.

Peng, Y., Sun, T. and Zhang, Y. (2017) Perception of salicylic acid in Physcomitrella patens. Front. Plant Sci. 8: 2145.

Pieterse, C.M.J., Leon-Reyes, A., Van der Ent, S. and Van Wees, S.C.M. (2009) Networking by small-molecule hormones in plant immunity. Nat. Chem. Biol. 5: 308-316.

Polischuk, V., Budzanivska, I., Shevchenko, T. and Oliynik, S. (2007) Evidence for plant viruses in the region of Argentina Islands, Antarctica: plant virus detection in Antarctica. FEMS Microbiol. Ecol. 59: 409-417.

Ponce de León, I. (2011) The moss Physcomitrella patens as a model system to study interactions between plants and phytopathogenic fungi and oomycetes. J. Pathog. 2011: 1-6.

Ponce de León, I., Hamberg, M. and Castresana, C. (2015) Oxylipins in moss development and defense. Front. Plant Sci. 6: 483.

Ponce de León, I. and Montesano, M. (2013) Activation of defense mechanisms against pathogens in mosses and flowering plants. Int. J. Mol. Sci. 14: 3178-3200.

Ponce de León, I. and Montesano, M. (2017) Adaptation mechanisms in the evolution of moss defenses to microbes. Front. Plant Sci. 8: 366.

Ponce de León, I., Oliver, J., Castro, A., Gaggero, C., Bentancor, M. and Vidal, S. (2007) Erwinia carotovora elicitors and Botrytis cinerea activate defense responses in Physcomitrella patens. BMC Plant Biol. 7: 52.

Ponce de León, I., Schmelz, E.A., Gaggero, C., Castro, A., Álvarez, A. and Montesano, M. (2012) Physcomitrella patens activates reinforcement of the cell wall, programmed cell death and accumulation of evolutionary conserved defence signals, such as salicylic acid and 12-oxo-phytodienoic acid, but not jasmonic acid, upon Botrytis cinerea infection: $P$. patens defence responses against B. cinerea. Mol. Plant Pathol. 13: 960-974.

Pressel, S., Bidartondo, M.I., Ligrone, R. and Duckett, J.G. (2010) Fungal symbioses in bryophytes: new insights in the twenty first century. Phytotaxa 9: 238.

Pressel, S., Ligrone, R., Duckett, J.G. and Davis, E.C. (2008) A novel ascomycetous endophytic association in the rhizoids of the leafy liverwort family, Schistochilaceae (Jungermanniidae, Hepaticopsida). Am. J. Bot. 95: 531-541.

Puttick, M.N., Morris, J.L., Williams, T.A., Cox, C.J., Edwards, D., Kenrick, P., et al. (2018) The interrelationships of land plants and the nature of the ancestral embryophyte. Curr. Biol. 28: 733-745.e2.

Qin, X., Su, X., Luo, H., Ma, R., Yao, B. and Ma, F. (2018) Deciphering lignocellulose deconstruction by the white rot fungus Irpex lacteus based on genomic and transcriptomic analyses. Biotechnol. Biofuels 11: 58

Reboledo, G., del Campo, R., Alvarez, A., Montesano, M., Mara, H. and Ponce de León, I. (2015) Physcomitrella patens activates defense responses against the pathogen Colletotrichum gloeosporioides. Int. J. Mol. Sci. 16: 22280-22298.

Rensing, S.A. (2017) Why we need more non-seed plant models. New Phytol. 216: $355-360$.

Rensing, S.A. (2018) Great moments in evolution: the conquest of land by plants. Curr. Opin. Plant Biol. 42: 49-54.

Rensing, S.A., Lang, D., Zimmer, A.D., Terry, A., Salamov, A., Shapiro, H., et al. (2008) The Physcomitrella genome reveals evolutionary insights into the conquest of land by plants. Science 319: 64-69.

Russell, J. and Bulman, S. (2004) The liverwort Marchantia foliacea forms a specialized symbiosis with arbuscular mycorrhizal fungi in the genus Glomus. New Phytol. 165: 567-579.

Seo, S., Okamoto, M., Seto, H., Ishizuka, K., Sano, H. and Ohashi, Y. (1995) Tobacco MAP kinase: possible mediator in wound signal transduction pathways. Science 270: 1988-1992.

Shimamura, M. (2016) Marchantia polymorpha: taxonomy, phylogeny and morphology of a model system. Plant Cell Physiol. 57: 230-256. 
Silvani, V.A., Rothen, C.P., Rodríguez, M.A., Godeas, A. and Fracchia, S. (2012) The thalloid liverwort Plagiochasma rupestre supports arbuscular mycorrhiza-like symbiosis in vitro. World J. Microbiol. Biotechnol. 28: 3393-3397.

Spoel, S.H. and Dong, X. (2008) Making sense of hormone crosstalk during plant immune responses. Cell Host Microbe 3: 348-351.

Spoel, S.H., Johnson, J.S. and Dong, X. (2007) Regulation of tradeoffs between plant defenses against pathogens with different lifestyles. Proc. Natl. Acad. Sci. USA 104: 18842-18847.

Spoel, S.H., Koornneef, A., Claessens, S.M.C., Korzelius, J.P., Van Pelt, J.A., Mueller, M.J., et al. (2003) NPR1 modulates cross-talk between salicylateand jasmonate-dependent defense pathways through a novel function in the cytosol. Plant Cell 15: 760-770.

Spribille, T. (2018) Relative symbiont input and the lichen symbiotic outcome. Curr. Opin. Plant Biol. 44: 57-63.

Strullu-Derrien, C. (2018) Fossil filamentous microorganisms associated with plants in early terrestrial environments. Curr. Opin. Plant Biol. 44: $122-128$

Stumpe, M., Göbel, C., Faltin, B., Beike, A.K., Hause, B., Himmelsbach, K., et al. (2010) The moss Physcomitrella patens contains cyclopentenones but no jasmonates: mutations in allene oxide cyclase lead to reduced fertility and altered sporophyte morphology. New Phytol. 188: 740-749.

Sugano, S.S., Nishihama, R., Shirakawa, M., Takagi, J., Matsuda, Y., Ishida, S., et al. (2018) Efficient CRISPR/Cas9-based genome editing and its application to conditional genetic analysis in Marchantia polymorpha. PLoS One 13: e0205117.

Thaler, J.S., Humphrey, P.T. and Whiteman, N.K. (2012) Evolution of jasmonate and salicylate signal crosstalk. Trends Plant Sci. 17: 260-270.

Thomma, B.P.H.J., Eggermont, K., Penninckx, I.A.M.A., Mauch-Mani, B., Vogelsang, R., Cammue, B.P.A., et al. (1998) Separate jasmonate-dependent and salicylate-dependent defense-response pathways in Arabidopsis are essential for resistance to distinct microbial pathogens. Proc. Natl. Acad. Sci. USA 95: 15107-15111.

Tsuda, K. (2018) Division of tasks: defense by the spatial separation of antagonistic hormone activities. Plant Cell Physiol. 59: 3-4.

Tsuneda, A., Chen, M.H. and Currah, R.S. (2001) Characteristics of a disease of Sphagnum fuscum caused by Scleroconidioma sphagnicola. Can. J. Bot. 79: $1217-1224$

Upson, J.L., Zess, E.K., Białas, A., Wu, C. and Kamoun, S. (2018) The coming of age of EvoMPMI: evolutionary molecular plant-microbe interactions across multiple timescales. Curr. Opin. Plant Biol. 44: 108-116.

Verhage, A., van Wees, S.C.M. and Pieterse, C.M.J. (2010) Plant immunity: it's the hormones talking, but what do they say? Plant Physiol. 154: 536-540.

Vigneron, N., Radhakrishnan, G.V. and Delaux, P.-M. (2018) What have we learnt from studying the evolution of the arbuscular mycorrhizal symbiosis? Curr. Opin. Plant Biol. 44: 49-56.

de Vries, S., de Vries, J., von Dahlen, J.K., Gould, S.B., Archibald, J.M., Rose, L.E., et al. (2018) On plant defense signaling networks and early land plant evolution. Commun. Integr. Biol. 11: 1-14.

White, T.J., Bruns, T., Lee, S. and Taylor, J. (1990) Amplification and direct sequenceing of fungal ribosomal RNA genes for phylogienetics. In PCR Protocols: A Guide to Methods and Applications. Edited by Innis, M. A., Gelfand, D. H., Sninsky, J. J. and WHite, T. J. pp. 315-322. Academic Press, San Diego.

Xue, J.-Y., Wang, Y., Wu, P., Wang, Q., Yang, L.-T., Pan, X.-H., et al. (2012) A primary survey on bryophyte species reveals two novel classes of nucleotide-binding site (NBS) genes. PLoS One 7: e36700.

Yamamoto, Y., Ohshika, J., Takahashi, T., Ishizaki, K., Kohchi, T., Matusuura, $\mathrm{H}$., et al. (2015) Functional analysis of allene oxide cyclase, MpAOC, in the liverwort Marchantia polymorpha. Phytochemistry 116: 48-56.

Yao, M., Li, W., Duan, Z., Zhang, Y. and Jia, R. (2017) Genome sequence of the white-rot fungus Irpex lacteus F17, a type strain of lignin degrader fungus. Stand. Genomic Sci. 12: 55. 